\title{
Derivation of Second Order Partial Differential Equation Indicating Wave and Heat Equation through the Use of the Navier Stoke's Equation for Unsteady and Incompressible Flow
}

\author{
N. Akhtar and M. G. A. Hayder Chowdhury
}

\begin{abstract}
In this paper, we have tried to approach the concepts of two-dimensional wave equation and one dimensional heat equation through the means of the Navier Stoke's equation for unsteady and incompressible flow. Our pursuit to do so has been supported with ample justifications and analytic discussions. The strong relation shared by the fluid dynamics, wave mechanics and heat flow has been brought to light through our attempts.
\end{abstract}

Index Terms - Navier Stoke's equation for incompressible flow;one dimensional heat equation;two dimensional wave equation;unsteady motion.

\section{INTRODUCTION}

Wave equation can be construed as one of the most important and fundamental equations of mechanics. It can also be interpreted meticulously in terms of Fluid Dynamics. In terms of physical interpretation, wave equations basically give insights on the propagation of oscillations at a fixed speed in some determined quantity. Let us assume that we have an array of little weights of mass m interconnected with massless springs of length $\mathrm{h}$ and spring constant $\mathrm{k}$. We are going to delve deeper into the concept to derive the second order partial differential equation of wave (also known as the 2D wave equation) with the means of Navier Stoke's equation for unsteady and incompressible flow.

The above derivation is going to be followed by the derivation of heat equation using the Navier Stoke's equation for unsteady and incompressible flow. According to the second law of thermodynamics, given there are adjacent bodies, heat will flow from the warmer body to the relatively less warm or colder body. This flow will be proportional to the difference of temperature and the thermal conductivity of the material between them.

Upon further analysis and deep-rooted comprehensions of this law, heat equation appears in the picture; its interpretation can be calibrated in the following manner: the rate at which a material at a point will absorb or give out heat is proportional to how much warm or cold the surrounding of that material is.

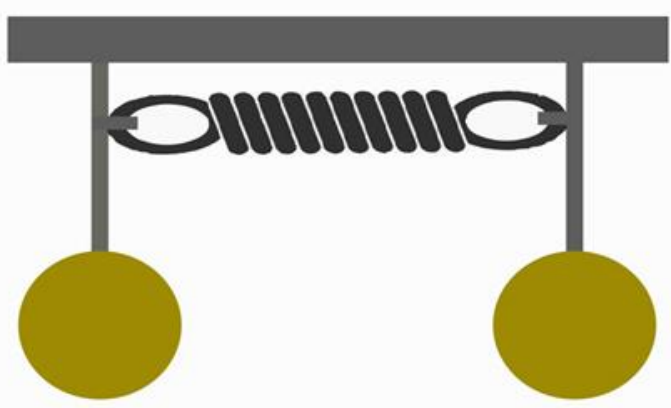

Fig. 1. Two masses connected with spring

\section{GOVERNING EQUATION}

A.

$\rho \frac{D u_{i}}{D t}=\rho F_{i}-\frac{\partial p}{\partial x_{i}}+\mu\left[\frac{\partial^{2} u_{i}}{\partial x_{j} \partial x_{j}}+\frac{1}{3} \frac{\partial \Delta}{\partial x_{i}}\right]$

This denotes the compressible Navier Stokes equation motion.

A case of great importance is that of an incompressible fluid when $\vec{\nabla} \cdot \vec{u}=\Delta=0\left(\frac{\partial u_{i}}{\partial x_{i}}=0\right)$; this denotes equation of continuity.

In which case, we get:

$\rho \frac{D u_{i}}{D t}=\rho F_{i}-\frac{\partial p}{\partial x_{i}}+\mu\left[\frac{\partial^{2} u_{i}}{\partial x_{j} \partial x_{j}}\right]$

This denotes incompressible Navier Stokes equation of motion.

Now expanding the L.H.S of equation (2), we get:

$\frac{\partial u_{i}}{\partial t}+u_{j} \frac{\partial\left(u_{i}\right)}{\partial x_{j}}=\rho F_{i}-\frac{\partial p}{\partial x_{i}}+\mu\left[\frac{\partial^{2} u_{i}}{\partial x_{j} \partial x_{j}}\right]$

In vector notation, equation (3) reduces to the following:

$\rho\left\{\frac{\partial \vec{u}}{\partial t}+(\vec{u} \cdot \vec{\nabla}) \vec{u}\right\}=\rho \vec{F}-\vec{\nabla} P+\mu \nabla^{2} \vec{u}$

Now neglecting body force $\rho \vec{F}$ and pressure force $\vec{\nabla} P$,

M. G. A Hayder Chowdhury, Shahjalal University of Science and Technology, Sylhet, Bangladesh. (e-mail: gahc_mat@yahoo.com)
Published on July 05, 2021.

N. Akhtar, Shahjalal University of Science and Technology, Sylhet, Bangladesh.

(e-mail: nazneenakhtar2827@gmail.com) 
inertia term $(\vec{u} . \vec{\nabla}) \vec{u}$ we get:

$\rho \frac{\partial \vec{u}}{\partial t}=\mu \nabla^{2} \vec{u}$

This denotes unsteady equation of motion.

Here $\vec{u}=l \hat{\imath}+m \hat{\jmath}+n \hat{k}$ (here, $\mathrm{l}, \mathrm{m}, \mathrm{n}$ denote velocity components in $\mathrm{x}$ axis, $\mathrm{y}$ axis and $\mathrm{z}$ axis respectively).

Now applying Newton's law to a single dimension of the above motion, we get:

$F_{\text {Newton }}=m \cdot \vec{a}(t)=m \cdot \frac{\partial^{2}}{\partial t^{2}} l(x+h, t)$

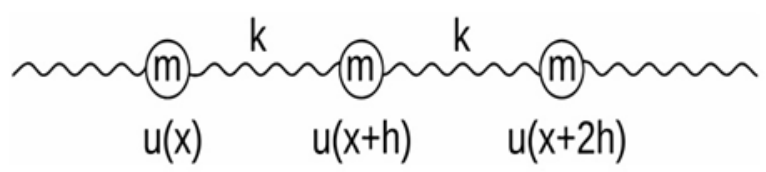

Fig. 2. Masses connected with a spring of spring constant $\mathrm{k}$.

Now, from equation 5 and equation 6 (here, we are considering $\mathrm{h}$ to be negligible )

We get $m=$ mass per unit volume

Therefore, $m=\rho$.

$m \frac{\partial^{2} l}{\partial t^{2}}=\nabla^{2} l$

Now applying the conversion of polar form $\nabla^{2}=\frac{\partial^{2}}{\partial x^{2}}+\frac{\partial^{2}}{\partial y^{2}}$ which is the Laplacian operator.

$x=r \cos \theta$

$y=r \sin \theta$

$r=\sqrt{x^{2}+y^{2}}$

$\theta=\tan ^{-1} \frac{y}{x}$

$\frac{\partial}{\partial x}=\frac{\partial}{\partial r} \cdot \frac{\partial r}{\partial x}+\frac{\partial}{\partial \theta} \cdot \frac{\partial \theta}{\partial x}$

$\frac{\partial}{\partial x}=\cos \theta \frac{\partial}{\partial r}-\frac{\sin \theta}{r} \frac{\partial}{\partial \theta} ; \frac{\partial}{\partial y}=\sin \theta \frac{\partial}{\partial r}+\frac{\cos \theta}{r} \frac{\partial}{\partial \theta}$

Therefore,

$$
\vec{\nabla}=\underline{i}^{r} \frac{\partial}{\partial r}+\frac{1}{r} \underline{i}^{\theta} \frac{\partial}{\partial \theta}
$$

Where,

$\underline{i}^{r}=\cos \theta \underline{i}+\sin \theta \underline{j}$

$\underline{i}^{\theta}=-\sin \theta \underline{i}+\cos \theta \underline{j} ; \frac{\partial \underline{i}^{r}}{\partial \theta}=\underline{i}^{\theta} ; \frac{\partial \underline{i}^{\theta}}{\partial \theta}=-i^{r}$

Now,

$\vec{\nabla} \cdot \vec{\nabla}=\nabla^{2}=\frac{\partial^{2}}{\partial r^{2}}+\frac{1}{r} \frac{\partial}{\partial r}+\frac{1}{r^{2}} \frac{\partial^{2}}{\partial \theta^{2}}$
Now, applying the Laplacian operator in polar form:

$\nabla^{2} l=\frac{\partial^{2} l}{\partial r^{2}}+\frac{1}{r} \frac{\partial l}{\partial r}+\frac{1}{r^{2}} \frac{\partial^{2} l}{\partial \theta^{2}}$

$\frac{m}{\mu} \frac{\partial^{2} l}{\partial t^{2}}=\frac{\partial^{2} l}{\partial r^{2}}+\frac{1}{r} \frac{\partial l}{\partial r}+\frac{1}{r^{2}} \frac{\partial^{2} l}{\partial \theta^{2}}$

where $\frac{m}{\mu}=\frac{1}{c^{2}}$ where $\mathrm{c}$ is constant.

$\frac{1}{c^{2}} \frac{\partial^{2} l}{\partial t^{2}}=\frac{\partial^{2} l}{\partial r^{2}}+\frac{1}{r} \frac{\partial l}{\partial r}+\frac{1}{r^{2}} \frac{\partial^{2} l}{\partial \theta^{2}}$

This is commonly known as second order partial differential equation; it can be also denoted as two dimensional wave equation.

Here, polar coordinates lie within the range: $0 \leq r \leq$ $R, 0 \leq \theta \leq 2 \pi$.

\section{B. Heat Equation}

Now, we are going to derive heat equation from Navier Stokes equation for unsteady incompressible flow.

From (4) we get:

$\rho \frac{D \vec{u}}{D t}=\rho\left\{\frac{\partial \vec{u}}{\partial t}+(\vec{u} \cdot \vec{\nabla}) \vec{u}\right\}$

$=\rho \vec{F}-\vec{\nabla} p+\mu \nabla^{2} \vec{u}$

Now, we are neglecting body force, inertia, and pressure term, we get equation for unsteady equation motion.

$\rho \frac{\partial \vec{u}}{\partial t}=\mu \nabla^{2} \vec{u}$.

Now we are assuming one dimensional equation of motion where $u$ is a function of $x \& t$.

Here, let $\frac{\mu}{\rho}=\alpha$ (where $\alpha$ is constant) and neglecting vector from both sides, we get the following second order partial differential equation:

$\frac{\partial u}{\partial t}=\alpha \frac{\partial^{2} u}{\partial x^{2}}$

This is a well-known heat equation (where $\mathrm{u}$ is the temperature and $\alpha$ denotes the thermal diffusivity of the medium).

\section{HEAT TRANSFER}
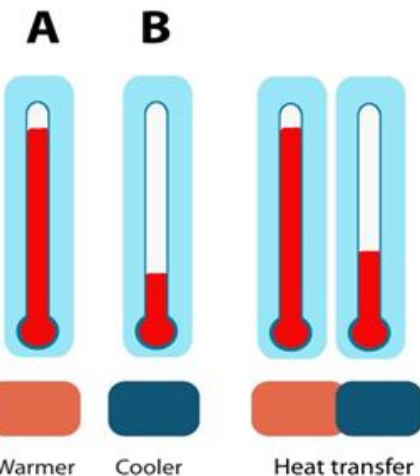

C

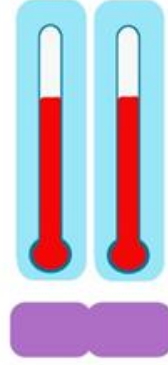

Heat transfer

Fig. 3. Heat flows from the warmer body to the cooler body. 


\section{CONCLUSION.}

Implementing the applications of Navier Stokes equation for unsteady and incompressible flow can interconnect fluid dynamics with other fields like hydrology, thermodynamics etc. Garnering sufficient insights on such relations can expand the knowledge levels and bring forth significant implications of the Navier Stokes equation. Delving deeper into such equations could result into meticulous findings that would embellish the aspects of fluid dynamics on a new level.

\section{ACKNOWLEDGMENT}

We are highly indebted to the contributions of the late Emeritus Professor Dr. J N Islam in fluid dynamics. His versatile work experiences and dutiful tutelage had been a great inspiration to us. He was an international scientist whose diligence towards science had been a massive success in various research fields.

\section{REFERENCES}

[1] G. K. Batchelor, Fluid Dynamics, Cambridge University Press, 1983, pp 143

[2] D. J. Triton, (Van Nostrand Reinhold (UK) Co. Ltd), Physical Fluid dynamics,

[3] Joseph H. Spurk, Fluid Mechanics, p101-102, Germany.

[4] Snneddon, Partial Differential equations.

[5] Piaggio, H. T. H, Differential equations.

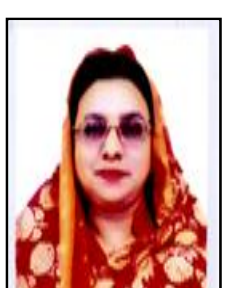

N. Akhtar was born in Chittagong. She studied in Dhaka University and received her B.Sc. Honors and Master's degree 19941995 from there. She accrued her $\mathrm{PhD}$ degree in the year 2008 from the RCMPS under the supervision of the Emeritus Professor $\mathrm{J} \mathrm{N}$ Islam, an International scientist. Her research field is mainly Fluid Dynamics.

She is currently working as a Professor in the Department of Mathematics, at Shahjalal University of Science and Technology, Sylhet, Bangladesh. She has published many articles in different renowned journals. Her research interests evolve around fields related to Fluid Dynamics.

Professor Dr. Akhtar is a lifelong member of the Bangladesh Mathematics Society.

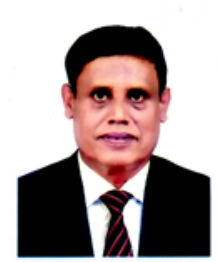

M. G. A Hayder Chowdhury was born in Golapganj, Sylhet. He studied in Chittagong University and received his B.Sc. Honors (1987) and Master's degree (1988) from there. He also attained the Chancellors award along with Talent pool Scholarship for being first class first in the B.Sc. Honors in the year 1987. He completed his MPhil degree 1992 and accrued PhD degree 2001 from the RCMPS under the supervision of the Emeritus Professor J N Islam, an International scientist. The author's research field is mainly fluid dynamics.

$\mathrm{He}$ is currently working as a Professor in the Department of Mathematics at Shahjalal University of Science and Technology, Sylhet, Bangladesh. He is also the current Head of the Department of Mathematics at SUST. He has published many articles in different renowned journals. His research interests mainly consist of fields related to Fluid Dynamics.

Professor Dr. Chowdhury is a lifelong member of the Bangladesh Mathematics Society. 